This item was submitted to Loughborough's Research Repository by the author.

Items in Figshare are protected by copyright, with all rights reserved, unless otherwise indicated.

\title{
Deformation analysis of aircraft wheels using a speckle shearing interferometer
}

PLEASE CITE THE PUBLISHED VERSION

PUBLISHER

(C) IMechE / Professional Engineering Publishing

LICENCE

CC BY-NC-ND 4.0

REPOSITORY RECORD

Ibrahim, Jamal S., Jon N. Petzing, and John R. Tyrer. 2019. "Deformation Analysis of Aircraft Wheels Using a Speckle Shearing Interferometer”. figshare. https://hdl.handle.net/2134/3779. 
This item was submitted to Loughborough's Institutional Repository (https://dspace.lboro.ac.uk/) by the author and is made available under the following Creative Commons Licence conditions.

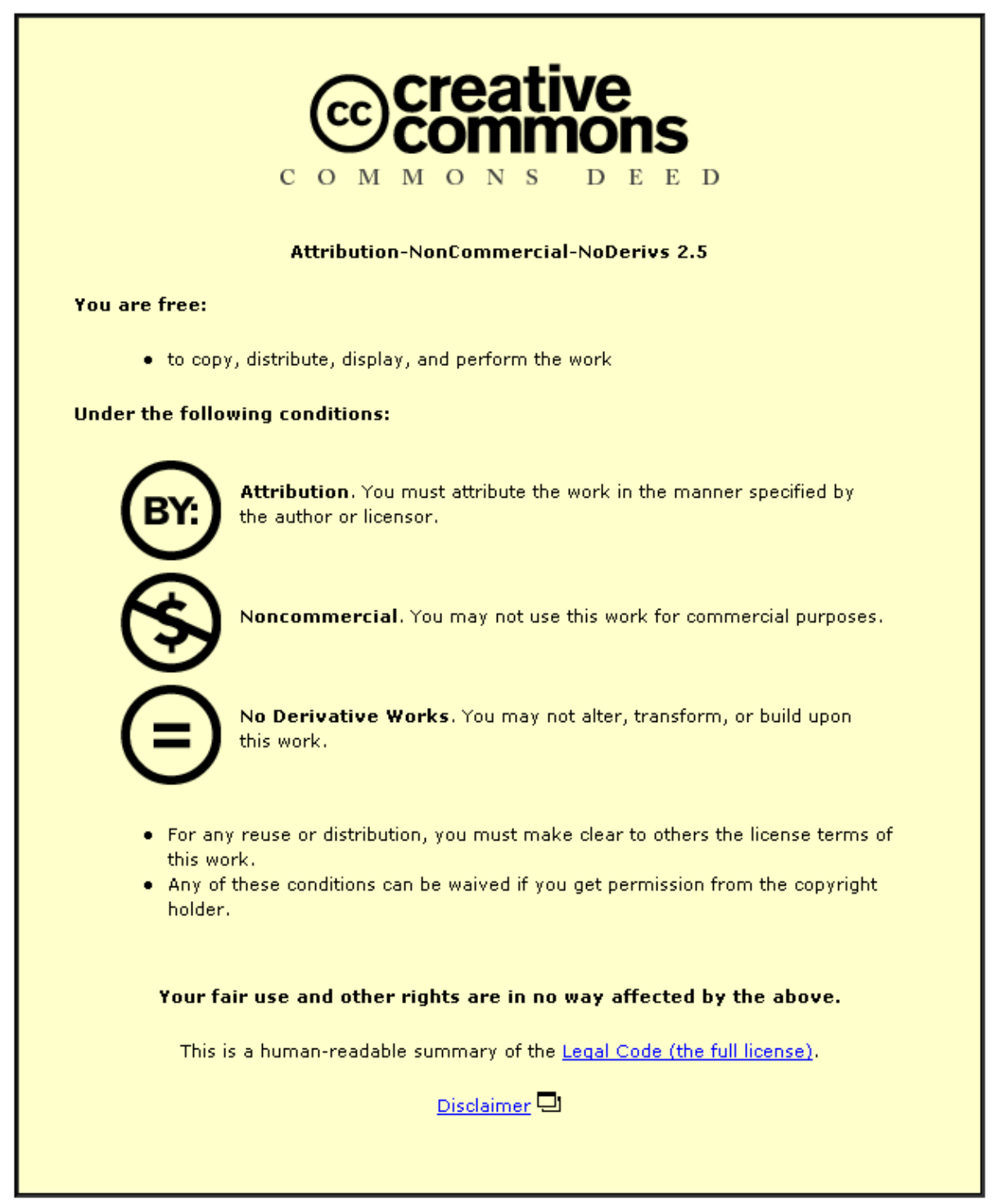

For the full text of this licence, please go to: http://creativecommons.org/licenses/by-nc-nd/2.5/ 


\title{
Deformation analysis of aircraft wheels using a speckle shearing interferometer
}

\author{
J S Ibrahim, J N Petzing* and J R Tyrer \\ Wolfson School of Mechanical and Manufacturing Engineering, Loughborough University, Loughborough, UK
}

\begin{abstract}
Speckle shearing interferometry has developed over the last three decades and is used as a non-destructive analysis tool for aerospace engineering. The primary role of the technique has traditionally been for the examination of defects in fibre composite structural materials, but more recent attention has considered strain and displacement measurement. Furthermore, aerospace regulatory bodies such as the UK Civil Aviation Authority are demanding better understanding and traceability of the technique.

The novel work reported here has investigated the use of a Michelson-based speckle shearing interferometer for the analysis of compression-loaded aircraft wheels and tyres. Whole-field deformation data have been obtained for repeated compression loading of a BAe146 main wheel, providing displacement derivative and displacement data. The quality of the measurement technique has been ascertained through the analysis of the repeatability and reproducibility data, with correlating data being produced using contact displacement transducers.
\end{abstract}

Keywords: aircraft, wheels, tyres, non-destructive testing, metrology, optical method, speckle shearing interferometry, deformation, displacement

\section{NOTATION}

$I_{\mathrm{A}} \quad$ image plane light intensity before deformation

$I_{\mathrm{B}} \quad$ image plane light intensity after deformation

$I_{1} \quad$ wavefront intensity from first mirror

$I_{2} \quad$ wavefront intensity from second mirror

$n \quad$ fringe order number

$\partial u / \partial x \quad$ in-plane first-order partial displacement derivative

$\partial w / \partial x \quad$ out-of-plane first-order partial displacement derivative

$\partial^{2} w / \partial x^{2} \quad$ out-of-plane second-order partial displacement derivative

$\partial w / \partial y \quad$ out-of-plane first-order partial displacement derivative

$x \quad$ Cartesian coordinate point reference

$X \quad$ Cartesian coordinate system axis

$y \quad$ Cartesian coordinate point reference

$Y \quad$ Cartesian coordinate system axis

$z \quad$ Cartesian coordinate point reference

$Z \quad$ Cartesian coordinate system axis

The MS was received on 5 November 2003 and was accepted after revision for publication on 17 June 2004.

* Corresponding author: Department of Mechanical Engineering, Loughborough University, Loughborough, Leicestershire, LE11 3TU, UK.e-mail:j.petzing@lboro.ac.uk

$\begin{array}{ll}\delta x & \text { small lateral shear in the } X \text { axis } \\ \delta y & \text { small lateral shear in the } Y \text { axis } \\ \delta \alpha & \text { small angular tilt } \\ \theta & \text { laser illumination angle } \\ \lambda & \text { laser wavelength } \\ \sigma & \text { standard deviation } \\ \phi & \text { optical phase change caused by deformation } \\ \psi & \text { initial optical phase }\end{array}$

\section{INTRODUCTION}

The manufacture of aircraft wheels requires quality control procedures and mechanisms to identify potentially defective units, during manufacturing and testing stages and operational service life. Defects can be formed due to random or systematic errors within the manufacturing processes and may cause problems such as fatigue fractures within the wheel structure. Use of such defective wheel and tyre units by an aircraft operator leads to growth of the defects, which in turn can potentially generate sudden unexpected catastrophic failure during flight-landing operations.

Qualification and approval of all wheel units is mandatory under UK Civil Aviation Authority 
(UKCAA) and Federal Aviation Administration (FAA), USA, regulations $[\mathbf{1 , 2}]$, as well as aircraft manufacturer internal standards. Hence, all wheel units are 100 per cent defect tested before customer delivery. The regulatory authorities and manufacturers have been requesting improvement and traceability of analysis methods, during manufacture and during routine inservice maintenance checks, linked through to the certification of testing systems and the operating personnel [3].

One particular analysis method which has seen growing acceptance within the aerospace industries as a useful analysis tool is speckle shearing interferometry (or shearography) [4], this technique being recognized under an FAA Advisory Circular [5]. The technique is a laser-based non-contact optical method, originally proposed in the early 1970s [6] and developed by a number of different optical engineering research groups during the 1970s [7] and 1980s [8]. Speckle shearing interferometry has now found some significant niche applications, specifically that of fault detection in fibre composites, and quality control of aircraft tyres.

The first application area has been the subject of recent UK [9] and European research contracts [10, 11], while other researchers are currently investigating the application of speckle shearing interferometry to rotating aircraft components [12]. The exploitation of much of this applied research has been in the form of commercial speckle shearing instrumentation. Further examples of application analysis of speckle shearing interferometry has been completed by The National Aeronautics and Space Administration (NASA) with reports available on the NASA Technical Reports Server; however, specific examples of the application of speckle shearing interferometry are available in the literature $[13,14]$.

In the case of the latter example of aircraft tyre analysis, equipment used to be based on photographic film, such as the former Grant Engineering Inc. L-RAY system. More recently, with the development of camera and image-processing technology, digital speckle shearing interferometry equipment such as the Steinbichler Optotechnik GmbH Intact ${ }^{(\mathbb{}}$ and the LTI Inc. ASTseries shearography tyre casing analyser now demonstrate the availability of the analysis technique. This is regarded somewhat as a mature technology with optical systems identifying and locating a number of different types of tyre defect during partial pressure tests.

A common aspect of much of this technology is the growing demand for quantitative data. Using imageprocessing algorithms and techniques [15] it is routine for a speckle interferometer to produce quantified whole-field meshes of deformation data. However, there is a increasing issue of the reliability of the data generated from speckle shearing interferometers. This concern is highlighted by a mounting body of evidence from a number of independent sources [16-20] over the last few years which have analysed aspects of the optical physics of the instrument, in order to determine sources of error which contribute to the final data values. What is clear from this work is that there are some specific optical design features (some of which have only recently come to light) which must be included within the instrumentation, in order to maximize the repeatability, reproducibility and consequently the reliability of the measurement method and the consequential data.

This paper examines the novel use of speckle shearing interferometry for aircraft component analysis, and specifically structural loading of aircraft wheels. The work considers the speckle shearing interferometer as a complete instrument and uniquely identifies the engineering reality of the repeatability and reliability of the technique, both in the controlled environment of the metrology laboratory, and within the industrial workplace. This work provides further evidence concerning the quality and strictures of the optical technique and may be of use when considering the complex issues of technique certification for aerospace structural analysis, and training issues for operatives.

\section{SPECKLE SHEARING THEORY}

Speckle shearing interferometers can be divided into groups, depending upon the optical method by which the spatial derivative data is produced. The Michelson shearing interferometer (shown schematically in Fig. 1) is one of several designs which use optical elements to split an incoming laser wavefront into two components, subsequently focusing these on to the image plane of the recording system $[\mathbf{4}, \mathbf{6}-\mathbf{8}]$.

Mirrors are placed parallel to two adjacent sides of the 50-50 non-polarizing amplitude beam-splitting cube, while a camera system views the laser illuminated object via the cube and mirrors. Spatial differentiation is controlled by the amount of lateral displacement or shear $(\delta x$ or $\delta y)$ created between the two wavefronts. This optical set-up is one of two common designs in commercial equipment, due to the ability to change the instrumentation sensitivity by altering the magnitude of the lateral shear. The alternative common commercial design uses an optical wedge, causing a fixed lateral shear determined by the wedge angle.

In the case of the Michelson interferometer, manipulation of the wavefront components is achieved using the mirrors (or prisms) positioned around the beamsplitting cube. Generally one mirror will be parallel to the respective cube face, while the other will have a small tilt $\delta \alpha$ introduced with respect to the second cube face. It is considered important to limit applied lateral shear to only one axis at a time when recording measurements. Shear in both the $X$ and the $Y$ axes is required to characterize fully the slope function of an object, but 


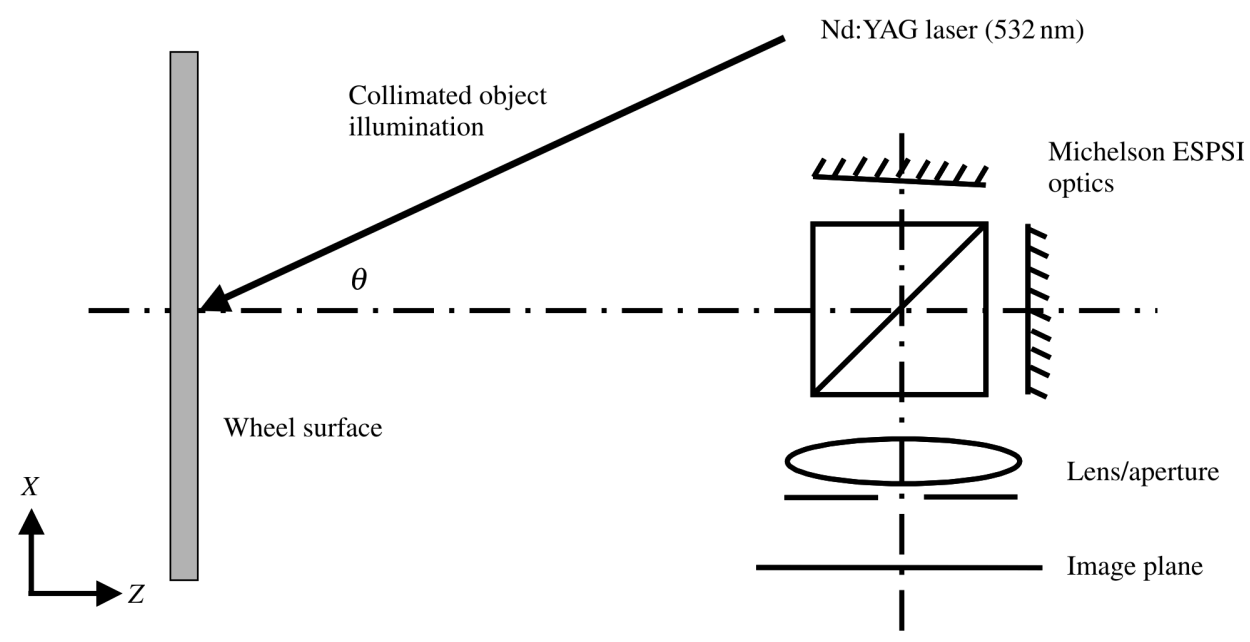

Fig. 1 Basic optical design of a speckle shearing interferometer (Nd: YAG, neodymium doped yttrium aluminium garnet; ESPSI, e\$\$ speckle shearing interferometry)

analysis of the data is difficult if multiple shears are used simultaneously.

The light intensity at the image plane can be described in a simplified form which is synonymous with speckle metrology:

$$
I_{\mathrm{A}}=I_{1}+I_{2}+2 \sqrt{I_{1} I_{2}} \cos \psi
$$

where the optical phase term $\psi=\theta(x+\delta x, y)-\theta(x, y)$, and $I_{1}$ and $I_{2}$ are the general intensity terms of the two wavefronts. Note that the descriptor $I_{\mathrm{A}}$ is used in this instance as a means of identifying the intensity distribution, attributable to the object in its initial state.

If the object receives a static deformation, the optical path alters slightly, represented as a phase change of $\phi(x, y)$ and $\phi(x+\delta x, y)$ respectively for the two image wavefronts created by the shearing optics. This creates a second set of equations which describe the object in its new state after the deformation function has been applied, which include the change in phase, and can be summarized as

$$
I_{\mathrm{B}}=I_{1}+I_{2}+2 \sqrt{I_{1} I_{2}} \cos (\psi+\phi)
$$

The change in intensity at the image plane can be described as $\Delta I=I_{\mathrm{A}}-I_{\mathrm{B}}$ and is typically achieved by storing an initial object state reference image and subtracting subsequent images from this reference, such that via trigonometric identities

$$
\Delta I=-4 \sqrt{I_{1} I_{2}} \sin \left(\psi+\frac{\phi}{2}\right) \sin \left(\frac{\phi}{2}\right)
$$

Examination of the relationship described in equation (3) leads to the conclusion that subtraction correlation fringe intensity (the raw data of the instrument) is at a maximum when $\phi=(2 n+1) \pi$, whereas minimum correlation fringe intensity occurs when $\phi=2 n \pi$, where, in both cases, $n$ is an integer number. It should be noted that equation (3) represents a single subtraction correlation interferogram, and phase-stepping techniques [15] are typically used to extract the optical phase in a quantified form.

Analysis of the optical path length changes within the interferometer can lead to a relationship that links the optical phase change and the spatial derivatives, in a form that is much used and referenced for common analysis of speckle pattern shearing correlation fringe patterns:

$$
\phi=\frac{2 \pi}{\lambda}\left[(1+\cos \theta) \frac{\partial w}{\partial x}+\sin \theta \frac{\partial u}{\partial x}\right] \delta x
$$

This relationship relates the optical phase to the partial displacement derivatives $\partial w / \partial x$ and $\partial u / \partial x$, where $\theta$ is the laser illumination angle with respect to the cameraimage plane axis. Equation (4) is specific to the illumination axis being in the $X Z$ plane and a horizontal lateral shear $\delta x$ with respect to the $X$ axis. Three further equations of a similar form may be developed when considering different orthogonal illumination vectors and lateral shear directions.

When examining an object for deformations typified by out-of-plane (OOP) signatures, then ideally this requires the illumination angle $\theta$ to be minimized, thus desensitizing the instrument with respect to in-plane terms $\partial u / \partial x$ and maximizing the OOP component sensitivity $\partial w / \partial x$. While the OOP slope component is of specific interest, it should be recognized that this term is not particularly significant with respect to engineering analysis and requires numerical integration to displacement, or further numerical differentiation before flexural strain $\partial^{2} w / \partial x^{2}$ can be derived. It is also important 
that the magnitude of the lateral shear $\delta x$ is small, because this has bearing upon the sensitivity of the shearing interferometer, the quality of the transducer signal and the inclusion of additional error terms [1719].

Using normal collimated illumination of the object, equation (4) can be reduced to the form

$$
\phi=\frac{4 \pi \delta x}{\lambda} \frac{\partial w}{\partial x}
$$

Assuming that data are produced in the form of subtraction correlation fringes, the OOP partial displacement derivative can be finally expressed as

$$
\frac{\partial w}{\partial x}=\frac{n \lambda}{2} \frac{1}{\delta x}
$$

Likewise, a similar relationship can be developed for the vertical OOP derivative component when using a small vertical shear $\delta y$ and illumination in the $Y Z$ plane:

$$
\frac{\partial w}{\partial y}=\frac{n \lambda}{2} \frac{1}{\delta y}
$$

Care must be taken when using these relationships as the basis of data processing, because they only hold true under certain conditions, these being collimated illumination of the object, viewing along the camera optical axis of the instrument and small shear values. Other work $[\mathbf{1 9}, \mathbf{2 0}]$ has already identified that equation (4) has very limited applicability (noted above) if quality and traceability of output are the priorities.

\section{EXPERIMENTAL DETAIL}

The interferometer design was based on the Michelson optics and is shown in Fig. 1. This type of speckle shearing interferometer was chosen because it provided sensitivity adjustment which was required in order to optimize the experimentation. However, it should be mentioned that the Michelson optics contain more air/ glass interfaces than a simple wedge-based system, resulting in a poorer system efficiency. The specific optical design of the instrumentation was constrained by dominating issues: firstly, the layout of the wheel testing facilities at Dunlop Aerospace Braking Systems Limited and, secondly, the requirement for collimated illumination, although every effort was made to consider and include instrument design features which acknowledge the existing body of knowledge concerning error sources and instrument performance.

The wheel units were illuminated using a continuouswave diode pumped neodymium-doped yttrium aluminium garnet, laser $(\lambda=532 \mathrm{~nm})$. The laser was expanded and collimated to $98 \mathrm{~mm}$ diameter, with the illumination angle $\beta$ being minimized to between $5^{\circ}$ and $7^{\circ}$ depending on object distance but could not be reduced further due to the optical arrangement. The instrument used a Pulnix TM9701D digital camera, linked through to a personal computer mounted Coreco Viper Digital frame-grabbing card. Image-grabbing and image-processing algorithms were developed and operated under the Coreco WIT and Sapera software packages. Two-, three- and four-step optical phase calculations [15] were implemented, used and evaluated. Final data processing was in Matlab, with optical phase extraction completed using PhaseVision Limited unwrapping software.

Whole-field maps of spatial derivatives were generated for all test conditions. Where appropriate, data were numerically integrated to produce specific displacement information. Sylvac P10 linear variable-displacement transducer (LVDT) displacement probes were used to provide pointwise correlating information, while Tekscan flexistrip pressure sensing units were used to monitor wheel/tyre interface pressures.

Wheel testing involved a BAe146 main wheel, with a Dunlop Aircraft Tyres Limited tyre, inflated to $160 \mathrm{lbf} /$ $\mathrm{in}^{2}$ using dry nitrogen. Wheel loading was completed in a load-cell-calibrated Denison compression-testing machine, specifically designed and configured for wheel loading, with Fig. 2 showing the loading system in a very simplified diagrammatic form. Compressive loading was completed in two parts: firstly, a preload of $10000 \mathrm{lbf}$ $(45.3 \mathrm{kN}), 15000 \mathrm{lbf}(67.95 \mathrm{kN})$ or $20000 \mathrm{lbf}(90.6 \mathrm{kN})$ was applied, which significantly reduced movement within the stub axle bearings; secondly, an incremental load of $500-2500 \mathrm{lbf}$ [in $500 \mathrm{lbf}(2.26 \mathrm{kN})$ steps] was applied in addition to the preload. Note that, due to the age of the equipment, all scales were marked in British Imperial units. Where appropriate, equivalent SI derived/related units were used and are provided for reference.

Experiments were designed and conducted to provide information to answer several issues:

1. Data were required to identify the repeatability/ reproducibility of the speckle shearing interferometer. This was achieved through an extensive series of repetitive loading experiments, by applying one preload and then repeatedly loading and unloading with small incremental loads.

2. Data were required to demonstrate the capability of the speckle shearing interferometer to measure whole-field wheel deformations while under large structural loads. This was achieved by measuring the deformation of the wheel while applying a variety of preloads and subsequent incremental loads, simultaneously generating correlating data with LVDT probes. It should be noted that applications of this nature (very large loading and analysis using speckle metrology) have received little if any attention within the engineering research literature. 


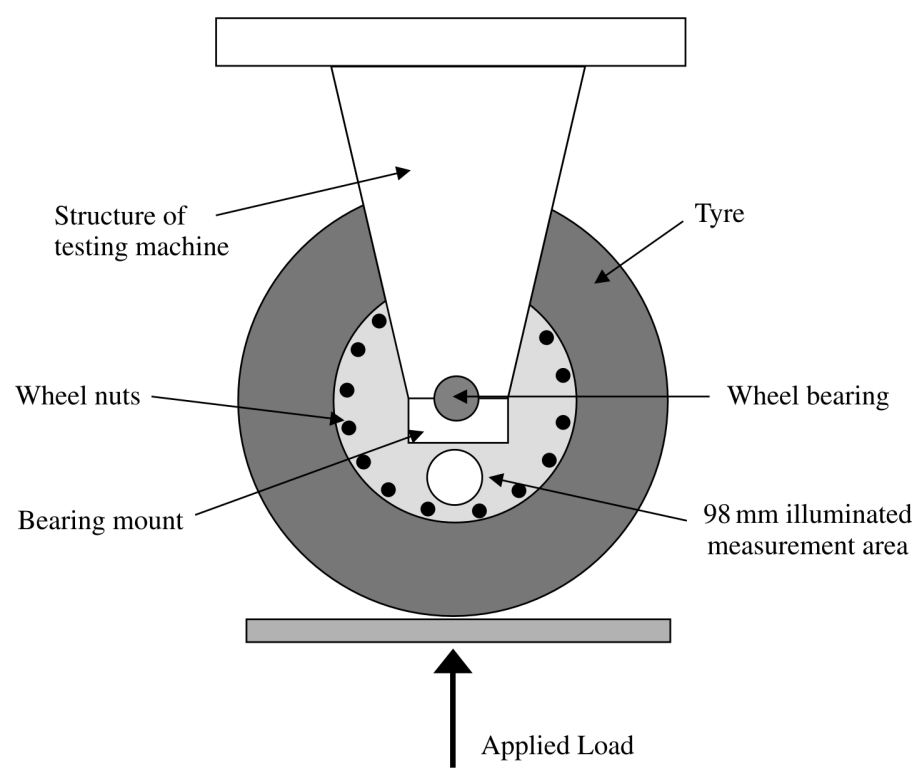

Fig. 2 Simplified schematic diagram of the compression loading test machine for aircraft wheels

3. Finally, data were required by other design elements of the aircraft wheel manufacturing company for the wheel design cycle, which was automatically a function of the two previous stages. However, owing to commercial confidentiality issues, wheel redesign is not discussed within this reported work.

\section{DISCUSSION OF EXPERIMENTAL RESULTS}

The experimental results are discussed in two parts. Firstly, it is demonstrated that the speckle shearing interferometer can produce meaningful engineeringbased numerical data from an aircraft wheel under simulated operational loads. Secondly, the quality and confidence of the data are discussed.

\subsection{Demonstrating the output of the instrumentation}

The primary output from a speckle shearing interferometer is a subtraction correlation fringe pattern, which depicts lines of isodisplacement derivative or isoslope. In reality, these are difficult to interpret manually or visually, and it requires automated extraction of the optical phase terms from the fringe patterns, in order to generate useful engineering information. This is a routine process within speckle metrology [15]. These automated stages are exemplified by Fig. 3 which shows the wrapped and unwrapped optical phase maps for the illuminated section of the BAe146 wheel of $98 \mathrm{~mm}$ diameter. The two small semicircular cut-outs at the bottom of each image in Fig. 3 are the positions of two consecutive wheel nuts, out of the total of 16 wheel nuts. The larger curved cut-outs at the upper sides of each

G05003 C IMechE 2004

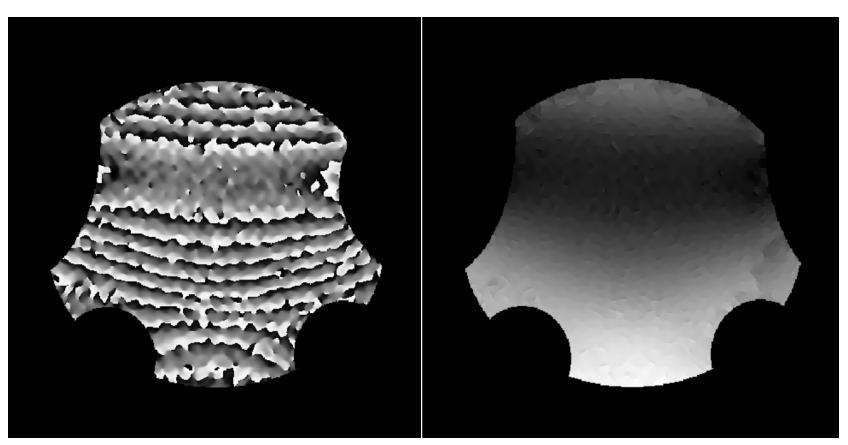

Fig. 3 Wrapped and unwrapped optical phase maps generated from the speckle shearing interferometer while testing the wheel/tyre under load

image in Fig. 3 are the position of wheel vent holes. These features have been blanked out during the analysis because data were not present in these regions.

The wrapped-phase map highlights an issue of data quality. In an ideal experimental situation, the grey-scale graduations between the black and white extremities of each wrapped optical phase fringe (left-hand image in Fig. 3) should be gradual, even and smooth [15]. In contrast, this data set contains discontinuities and nonsmooth data and is perhaps more typical of the quality of data obtainable in a non-controlled industrial context. Only moderate low-pass Fourier filtering was applied during the use of the speckle shearing interferometer. The reasons for this type of noise in the data is a function of different sources: structural vibration, local variations in object surface reflectivity, local variations in air refractive index, and instrument errors such as fringe contrast, speckle size and phase-stepping non-linearities. Removal of these noise terms is often 
desirable but may be problematic if using imageprocessing techniques, because the spatial frequencies of the noise terms overlap those of the speckle correlation data itself.

Depending on the type of phase-unwrapping algorithm used, then noise within the wrapped optical phase map may translate into the unwrapped map and consequently into three-dimensional data meshes. This is particularly the case when simple linear unwrapping routines, such as those supplied automatically in Matlab, are used. In this case, the research work included the prototyping of new unwrapping software (PhaseVision Limited) which automatically recognizes phase discontinuities caused by noise elements and significantly reduces noise transfer [21].

Calculating numerical data from the wrapped-phase map is a consequence of the application of equations (5) and (6), when considering the case of the $\partial w / \partial x$ terms. An example of the displacement derivative output is shown in Fig. 4, the data mesh being generated from the optical phase data shown in Fig. 3. Three issues of importance should be mentioned: firstly, that the displacement derivative data is dimensionless in terms of SI units, secondly, the instrumentation has been used to analyse the vertical OOP derivative components $\partial w / \partial y$ and, thirdly, for the purposes of clarity and presentation, the data mesh has been inverted. Hence the wheel nut positions are now represented by the two semicircular cut-outs at the top of the mesh.

Displacement data can be produced via numerical integration and are demonstrated in Fig. 5. The data show the surface of the wheel deformed away from the instrument as a function of load. Typically, loads of 500

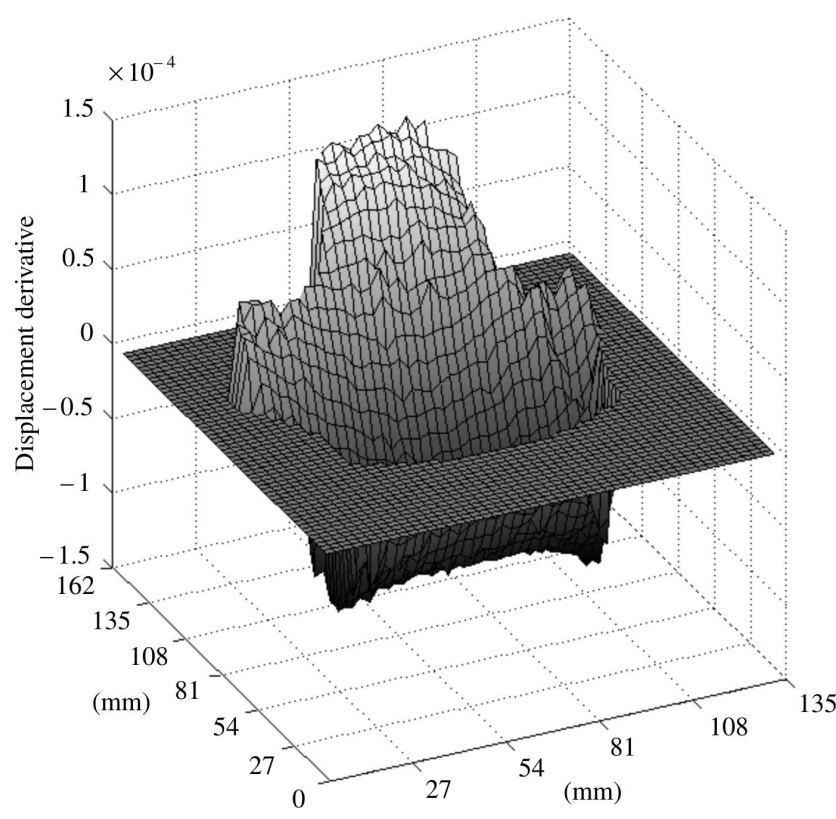

Fig. 4 Displacement derivative data $\partial w / \partial y$ calculated from the wrapped optical phase data

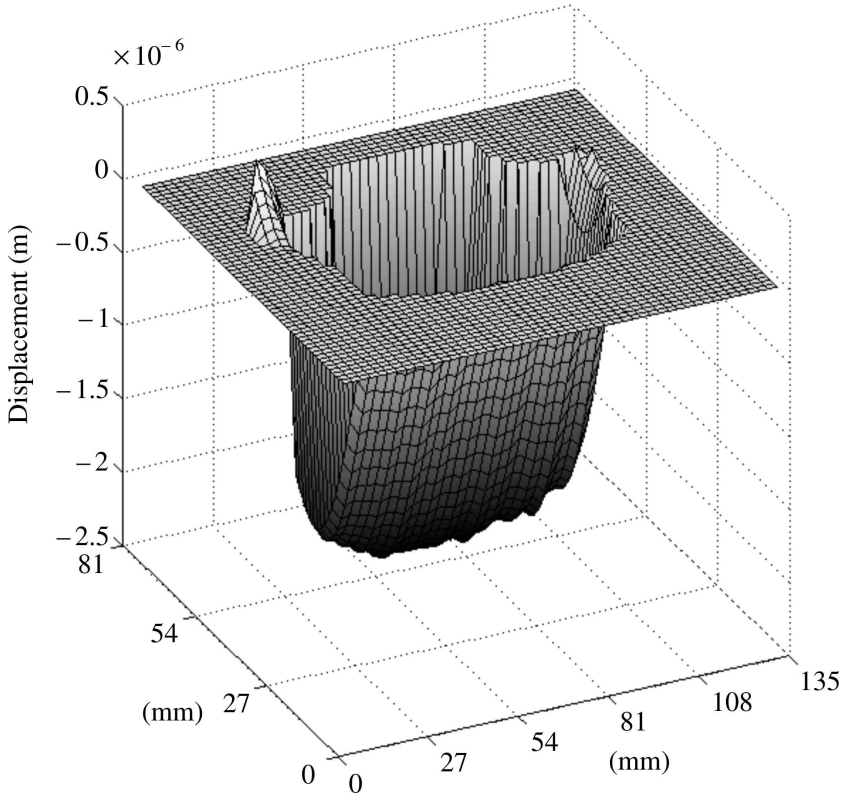

Fig. 5 Displacement data calculated from the displacement derivative data

to $2500 \mathrm{lbf}$ applied to the wheel (on top of the applied preload) caused surface displacement components of between 2 and $15 \mu \mathrm{m}$. Clearly, the deformation of the wheel as a function of the preloading would be significantly larger, but this was not measured due to movements of the wheel bearings. Furthermore, it was found that incremental loads larger than $4000 \mathrm{lbf}$ overloaded the sensitivity of the instrumentation. Sensitivity adjustment could be made to allow larger load analysis, but desensitizing speckle shearing interferometers tend to lead to poorer data contrast $[\mathbf{1 6}]$ and more noise in the numerical data.

Care must be taken when interpreting the data shown in Fig. 5, because it only represents the numerical integration of one of the two OOP displacement derivative components, namely $\partial w / \partial y$. The analysis for the other OOP component $\partial w / \partial x$ has not been presented here because the magnitude of the $\partial w / \partial x$ data was negligible in comparison with the $\partial w / \partial y$ data. However, when analysing other structural objects with different deformation characteristics, the $\partial w / \partial x$ term may be more significant. If so, then it would be necessary to measure and process both sets of deformation derivatives $\partial w / \partial x$ and $\partial w / \partial y$, numerically to integrate and then to combine, to obtain the correct representation of the OOP plane object surface displacement.

\subsection{Issues of data quality}

Defining the accuracy of a measurement instrument should normally involve a direct or indirect (via 


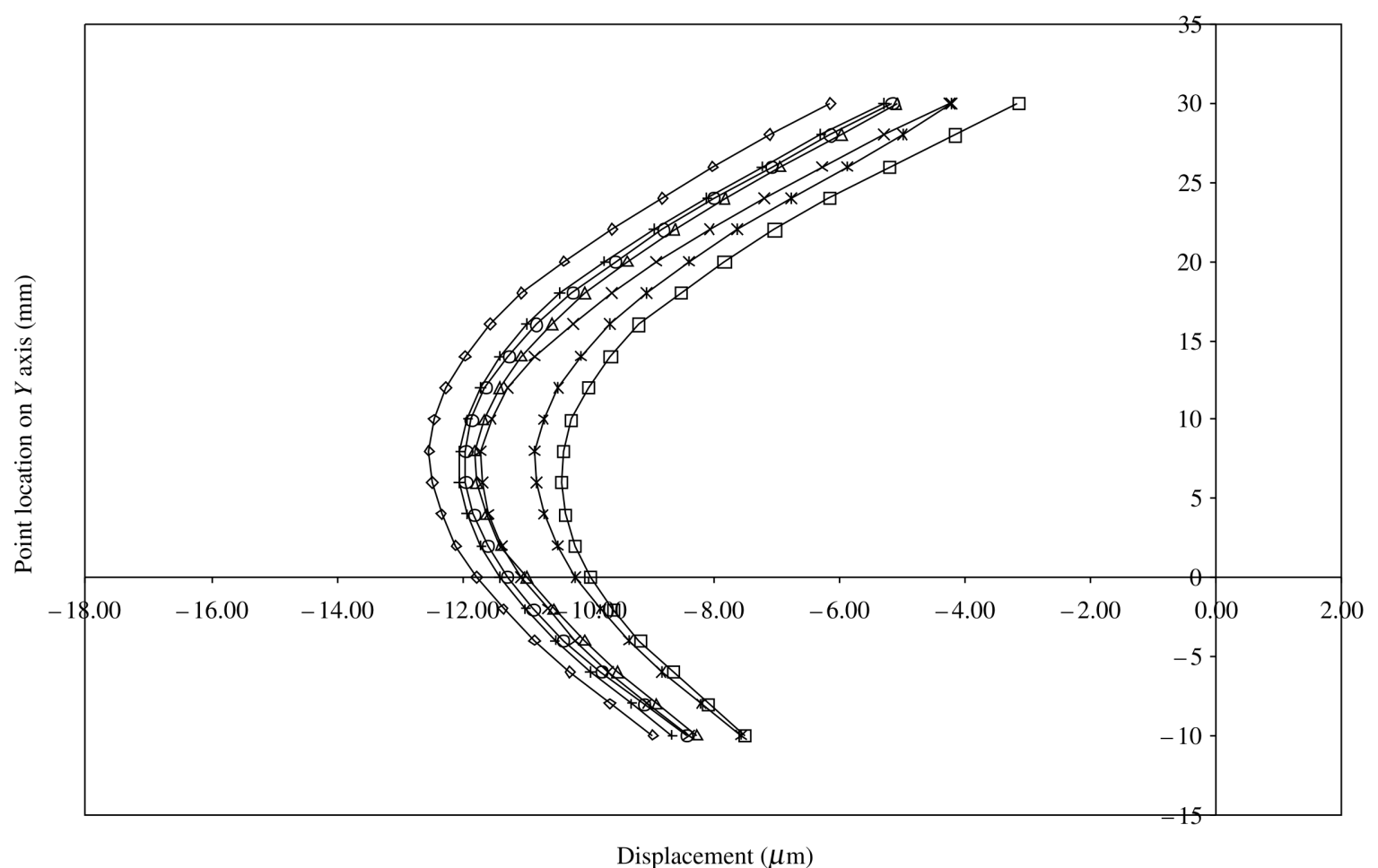

$24 / 02 / 03 \mathrm{am} \rightarrow-25 / 02 / 03 \mathrm{am} \triangle-25 / 02 / 03 \mathrm{pm} \rightarrow-26 / 02 / 03 \mathrm{am} \rightarrow-26 / 02 / 03 \mathrm{pm} \rightarrow-27 / 02 / 03 \mathrm{am} \rightarrow 27 / 02 / 03 \mathrm{pm}$

Fig. 6 Repeated line profiles of displacement taken through a vertical section of the wheel profile

working/transfer standards) comparison with the relevant primary standard. This is often achieved through comparison measurements, or the use of an artefact of known properties. Unfortunately, speckle shearing interferometers and other similar whole-field techniques do not currently have a route of traceability to the primary length standard, the metre, although a recently initiated European Union funded project [22] is considering these issues as its core topic area.

It is, however, possible to define the quality and confidence of the instrument and measurements through an understanding of the repeatability and reproducibility of the data. This has been completed by the present authors and has been demonstrated for one particular design of speckle shearing interferometer, ideal laboratory-based repeatability versus the reality of industrial measurement repeatability. This work is further extended by the results presented here, which demonstrates the repeatability of the speckle shearing interferometer for the displacement analysis. Figure 6 shows data obtained for a preload of $20000 \mathrm{lbf}$ and incremental load of $2500 \mathrm{lbf}$ on the BAe146 wheel. These data have been processed and developed to provide displacement component line profiles vertically through the analysis area of $98 \mathrm{~mm}$ diameter, from the $\partial w / \partial y$ partial derivative data.

The experimentation represented in the data within Fig. 6 was repeated on seven occasions, leading to seven line profiles of displacement component. It is noticeable that there is a variation with time, this possibly being caused by changing equipment usage (and consequently environmental noise sources) within the test environment, other than the equipment reported here. The variability of the changing noise sources introduces more uncertainty into the comparability of the data, but this is the reality of operating interferometric measuring equipment in industrial environments. In many cases, it will not be possible to control the environment, only the local test conditions and variables.

The operational set-up of the speckle shearing interferometer and the wheel testing equipment remained identical throughout the testing period, although it is recognized that issues of backlash, hysteresis, creep/relaxation in the rubber matrix of the tyre, and other machine errors may also have contributed to the variation. The data in Fig. 6 may be summarized by Fig. 7, which shows the mean data line, the $2 \sigma$ standard deviation bounds and data obtained from the LVDT.

The purpose of showing the $2 \sigma$ bounds is that they represent the 95 per cent confidence limits of the data. This leads to a maximum $2 \sigma$ value for the speckle shearing derived displacement data of $3.7 \mu \mathrm{m}$ for this particular work. The pointwise LVDT data tend to agree with the optical data, although the extent of the $1 \mu \mathrm{m}$ error bars on the LVDT data is significant. The 


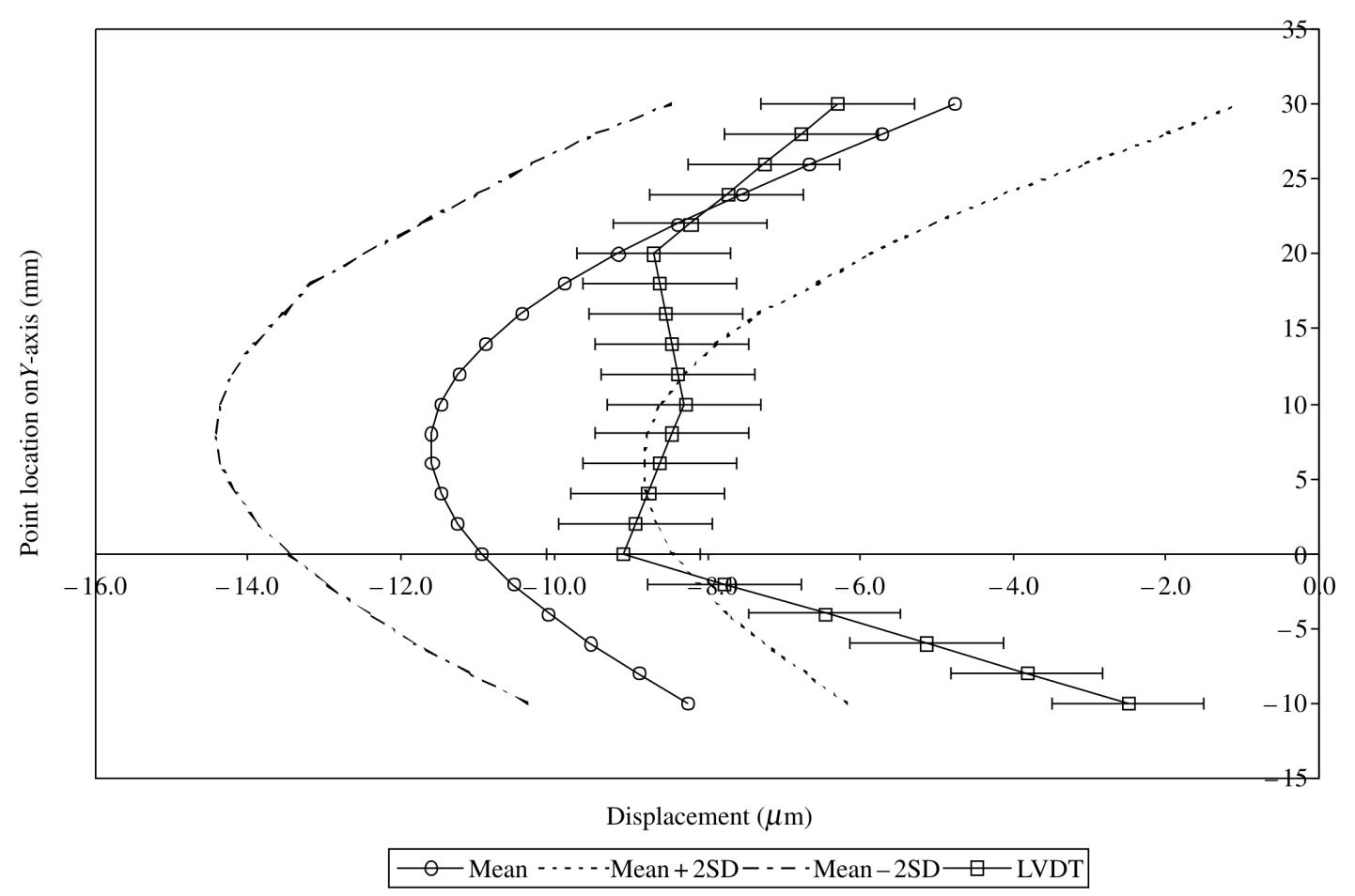

Fig. $72 \sigma$ repeatability bands and LVDT data, compared with the mean displacement profile derived from the speckle shearing interferometer

stated accuracy of the Sylvac P10 displacement transducer ( $10 \mathrm{~mm}$ range) is $1 \mu \mathrm{m}$ with a $0.1 \mu \mathrm{m}$ repeatability. However, the transducer was used to measure small displacements $(0-12 \mu \mathrm{ms})$, which is very close to its noise floor. Any transducer errors may therefore become more significant and will influence the correlation with the optical data. A simple example is if the transducer is not placed normal to the measured surface, thus causing a cosine error and an incorrect reading.

It is important to realize that this statement of displacement component measurement quality, in terms of repeatability (or reproducibility [23]), is specific to the design of the speckle shearing interferometer, the software and hardware specifications of the image processing, and the operational characteristics of the aircraft wheel compression-testing machine. If the measurement instrument is transferred to another applications area, then the experimental variables (and error sources) will be changed and potentially the repeatability may also change. An additional issue that should also be considered is the form of the object being analysed, because the relationships discussed in section 2 are particularly relevant to the analysis of planar (flat) surfaces. If the object being analysed is not planar, then the change in geometry will cause a change in instrument sensitivity, which if not taken into account will create further errors in the data processing and interpretation.

\section{CONCLUSIONS}

This paper has described the novel and successful application of speckle shearing interferometry to the analysis of large-scale, structurally loaded aircraft wheels. Specifically, a BAe146 main wheel has undergone a series of compression load tests, using a preload of between 10000 and $20000 \mathrm{lbf}$, with incremental loads of 500-2500 lbf. Whole-field non-contact analysis has identified the real-time deformation of the wheel structure. These data have been processed to produce displacement component data showing the surface deformations and have been obtained on a routine basis, in what can be described as an active industrial environment, with no form of vibration isolation.

Another important novel aspect of this work has been to consider the quality and confidence of the measurements. The shearing interferometer has measured surface displacement components in the range $0-15 \mu \mathrm{m}$, which correlates with pointwise data collected using an LVDT. Through repeated experimentation, the $2 \sigma$ displacement component repeatability of the instrument for this specific object/load case has been shown to be $3.7 \mu \mathrm{ms}$, although it is important to note that this value will change if the experimental conditions or the design of the optical transducer are changed.

As part of the discussion, the work has identified critical aspects of speckle shearing interferometer design 
and application, all of which will influence the quality and integrity of data produced. With respect to current UK CAA and FAA regulations, this work has generated evidence of data confidence, instrumentation design criteria and instrumentation procedure, which will be valuable within the aerospace (and related) sector. Finally, the work has demonstrated that the instrumentation can produce deformation data in standard engineering measurands which is suitable for design analysis verification.

\section{ACKNOWLEDGEMENTS}

The work reported has been completed under the auspices of the DTI/LINK Engineering and Physical Sciences Research Council sensors and sensor systems for industrial applications contract; 'Aircraft tyre examination using optical strain testing (AIRTEST)' (GR/ M78960), in association with Dunlop Aerospace Braking Systems Limited and Laser Optical Engineering Limited.

\section{REFERENCES}

1 Aircraft wheels and wheel-brake assemblies. Technical Standard Order TSO-C26c, Federal Aviation Administration, Washington, DC, 1984.

2 Tyres and wheels fitted to aircraft certificated in the transport category. Airworthiness Notice 93(5), UK Civil Aviation Authority, March 2002.

3 Personnel certification for non-destructive testing of aircraft, engines, components and materials. Airworthiness Notice 94(7), UK Civil Aviation Authority, March 1999.

4 Steinchen, W. and Yang, L. Digital Shearography: Theory and Application of Digital Speckle Pattern Shearing Interferometry, 2003 (Society of Photo-optical Instrumentation Engineers, Bellingham, Washington).

5 Acceptable methods, techniques and practices - aircraft inspection and repair. Advisory Circular AC 43.13-IB, Federal Aviation Administration, Washington, DC, 2001.

6 Leendertz, J. A. and Butters, J. N. An image shearing speckle pattern interferometer for measuring bending moments. J. Physics E, 1973, 6, 1107-1110.

7 Hung, Y. Y. and Taylor, C. E. Measurement of slopes of structural deflections by speckle shearing interferometry. Expl Mechanics, 1974, 14, 281-285.

8 Sirohi, R. S. Speckle Metrology, 1993 (Marcel Dekker, New York).
9 Huntley, J. M., Richardson, M. O. W. and Nurse, A. D. Insitu quantitative flow detection using advanced optical inspection techniques. Report (GR/M57835), Engineering and Physical Sciences Research Council, 2003.

10 Pulsed digital holography and shearography. BRITE/ EURAM 3 Report (BRPR 960152), EU Fourth Framework Programme, 1999.

11 Multi-wavelength shearography. BRITE/EURAM 3 Report (BRPR 960272), EU Fourth Framework Programme, 1999.

12 Irving, P. E., Tatam, R. P. and Thompson, C. P. Damage detection and damage tolerance in rotating mechanical systems. Report (GR/N11872), Engineering and Physical Sciences Research Council, 2003.

13 Parker, S. C. J. and Salter, P. L. A novel shearography system for aerospace non-destructive testing. Proc. Instn Mech. Engrs, Part G: J. Aerospace Engineering, 1999, 213(G1) 23-33.

14 Steinchen, W., Yang, L. and Mäckel, P. Non-destructive testing of aerospace composite materials using digital shearography. Proc. Instn Mech. Engrs, Part G: J. Aerospace Engineering, 1999, 212(G1) 21-30.

15 Robinson, D. W. and Reid, G. T. Interferogram Analysis: Digital Fringe Pattern Measurement Techniques, 1993 (Institute of Physics, Bristol).

16 Ng, T. W. and Chau, F. S. Statistical properties of phase variation in shearing speckle interferometry and its effect on fringe visibility. J. Mod. Optics, 1992, 39(12), 24632469.

17 Krishna Mohan, N., Masalkar, P. J., Murukeshan, V. M. and Sirohi, R. S. Separation of the influence of in-plane displacement in multiaperture speckle shear interferometry. Opt. Engng, 1994, 33(6), 1973-1982.

18 Steinchen, W., Yang, L., Kupfer, G., Mäckel, P. and Vössing, F. Strain analysis by means of digital shearography: potential, limitations and demonstration. J. Strain Analysis, 1998, 33(2), 171-182.

19 Wan Abdullah, W. S., Petzing, J. N. and Tyrer, J. R. Wavefront divergence: a source of error in quantified speckle shearing data. J. Mod. Optics, 2001, 48(5), 757-772.

20 Farrant, D. I. and Petzing, J. N. Sensitivity errors in interferometric deformation metrology. Appl. Optics, 2003, 42(28), 5634-5641.

21 Huntley, J. M. Automated fringe pattern analysis in experimental mechanics: a review. J. Strain Analysis, 1998, 33(2), 105-125.

22 Standardisation project for optical techniques of strain measurement. Report G6RD-CT-2002-00856 (SPOTS), EU Sixth Framework Programme, 2002.

23 PD 6461: Part 2 Vocabulary of Metrology: Vocabulary of Legal Metrology-Fundamental Terms, 1980 (British Standards Institution, London). 